\title{
The Factor Analysis and Preventive Measures of Ankle Injury in Basket- ball Players' Movement
}

\author{
Zhixiang $\operatorname{Jin}^{*}$
}

Department of Sports, Huazhong Agricultural University, Wuhan 430070, Hubei, China

\begin{abstract}
Basketball players are in the fast fierce competition for control ball and scoring process, because of the influence of internal and external factors and the rapid movement of the players on the limited site, sports damage is inevitable. This article will be through the literature method, questionnaire survey, data statistical analysis and logical analysis to find out the factors that affect the injury of ankle joint in basketball, study the law, reduce or avoid the injury of ankle joint in basketball. It is of great significance to improve the basketball sport level and prolong its sports life. Preventive measures are put forward to reduce the unnecessary damage in basketball and promote the development of basketball.
\end{abstract}

Keywords: Ankle injury, basketball movement, method, prevention.

\section{INTRODUCTION}

With the popularization of basketball, more and more people are involved in. However, the basketball technology continues to improve, and constantly challenge the physical limit, this situation makes the incidence of ankle injuries in basketball sports higher and higher. To strengthen the research on basketball ankle injury causes and prevention measures will not only improve the quality of sports population, in order to promote the development of school sports and community sports development, promote the national fitness and expand our sports population, promote the rapid spread of the sports knowledge and improve the consciousness of the whole people health has a larger role in promoting. Modern basketball as a global social culture, characterized by continuous to the high strength, high speed and strong against development, body, technology, intelligence, will and spirit of teamwork of the modern movement. Due to the basketball competition increasingly fierce, strong confrontation, offense and defense conversion speed and so on, so in basketball sports injury more frequently. Among them, sprain of ankle joint is the most common, mainly in lateral ankle ligament injury. And then including: lateral ankle ligament injury, medial ligament injury and cartilage damage of bone joint. The injury of ankle joint seriously affects the improvement of the technical level of the basketball players, and some even cause lifelong regrets. When the plantar flexion, the ankle joint has the activity degree, the muscle strength of the foot is bigger than the muscle group of the foot. The strength of the medial triangular ligament of the ankle is stronger than that of the anterior lateral ligament, which limits the ability of rapid inversion of the foot, and makes the pinning force weaker. When the foot to the others feet or uneven ground and make the body imbalance, weight

*Address correspondence to these authors at the Department of sports, Huazhong Agricultural University, Wuhan 430070, Hubei, China; E-mail: hmzdld159@sina.com falls to distort the soles of the feet, resulting in ankle lateral ligament injury, which is in the stadium occurred common examples of sports injury. The injury of ankle joint is common in basketball, and the lateral collateral ligament is the most common. If the early treatment is not good, it will seriously affect the training of athletes, and may cause serious sequela. According to relevant statistics show, $92 \%$ of basketball athletes had lateral ligament of ankle joint injury, 8 $3 \%$ had more than twice the damage; China's national team athletes in $73 \%$ had more than two times of the lateral ankle ligament injury, $59 \%$ residual all residual symptoms [1]. Therefore, the prevention of ankle injury is more important. Through to amateur sports school basketball athlete's ankle joint injury were investigated, and analysis of damage causes and mechanisms, in order to do a better job of prevention, to achieve the basketball exercise reduces the injury rates, to ensure normal training.

The ankle joint strain is a kind of disease evidence of ankle ligament injury or rupture. The injury can occur at any age, children of school age in a large number of activities. Modern medicine thinks, ankle joint is twisted much in walking, running, jumping or down stairs. Downhill, ankle plantar flexion and suddenly outward or inward, lateral or medial collateral ligament by strong tension, resulting in the stability of the ankle lose balance and harmony, and the incidence of ankle sprain. The lateral ankle injury is the most common.

\section{RESEARCH OBJECT AND METHOD}

\subsection{Research Object}

Object for Fuzhou Vocational and Technical College basketball enthusiasts, [2] Fujian Normal University, sports professional basketball special students, Fuzhou 170 basketball club players. The total number of 161 people, including 124 male and 37 female; the age ranged from 17 to 28 years old, the average age of 21 years; 4 to 6 years of age most. 
Table 1. Injury part of basketball players $(\mathrm{N}=141)$.

\begin{tabular}{|c|c|c|c|c|c|c|}
\hline Total Person Time & Ankle Joint & Finger & Knee & Waist & Foot & Leg \\
\hline \hline 188752 & $36.98 \%$ & $24.78 \%$ & $14.62 \%$ & $11.11 \%$ & $7.95 \%$ & $4.54 \%$ \\
\hline
\end{tabular}

Table 2. Damage parts of ankle joints.

\begin{tabular}{|c|c|c|c|}
\hline Total Person Time & Lateral ankle Ligament & Medial Ligament & $\begin{array}{c}\text { Avulsion Fracture of } \\
\text { Tibia and Fibula }\end{array}$ \\
\hline \hline 67920 & $56 \%$ & $7.1 \%$ & $3.54 \%$ \\
\hline
\end{tabular}

Fig (1). Injury part of basketball players $(\mathrm{N}=141)$.

\subsection{Research Methods}

- Literature Journal of physical education related materials and related news search data [3].

- Questionnaire design the questionnaire of the common injury in basketball, altogether provides questionnaire 161,144 questionnaires were retrieved, with the recovery rate of $89.4 \%$, the efficiency of $95 \%$, to recover the questionnaire for statistical data, processing and comprehensive analysis

- Telephone network interview, by telephone, QQ, e-mail tracking interviews, in order to obtain more information and data.

- Data statistics method will receive data from the Excel software to recover the questionnaire data statistics and processing results

\section{RESULTS AND ANALYSIS}

\subsection{The Parts Of The Basketball Sports Injury}

Of the 161 respondents, a total of 188752 people were injured. No significant differences between men and women, in the basketball sports injury of the parts of the questionnaire can be seen basketball 'ankle injury 35.98\% and finger injuries accounted for $25.78 \%, 14.62 \%$ of knee injury, waist injury $11.11 \%$, foot injury accounted for $7.95 \%$, accounting for $4.54 \%$ calf injury. Therefore, it is not difficult to see basketball player damage mainly to the ankle joint, accounting for one third of all the site of injury [4]. Fig. (1). As shown in Table 1.
In the ankle injury site survey found that altogether has ankle injury 67920 passengers, the lateral ankle ligament injury $56 \%$, followed by bone and joint cartilage injury accounted for $3.35 \%$, medial ligament injury to $7.1 \%$ and avulsion fracture of tibia and fibula was only $3.54 \%$ [5]. As shown in Table 2.

The ankle joint is the starter of human body bounce, also landing buffer device. First, we understand the structural features of ankle ligaments. Fork is composed of lower joint surface of tibia, the joint surface of the medial ankle and fibula ankle surfaces. Joint surface of the tibia bottom, and the medial ankle surface are connected into a whole, jointly with the talus is formed by connecting the ankle surface of talus in talus below is the biggest piece of bone. Tarsal back projection called tubercle, part of ankle motion in addition to the muscles but also have a non-negligible called the tendons and ligaments, there are three main groups [6]: (1) Medial ligament known as the "triangle ligament" is the toughest ankle ligaments main function is to prevent ankle, starting from the ankle, "fanned down" splitting beyond the bone. (2) Lateral ligament, lateral ankle and divided into three bundles were ended in the talus, lateral and talus behind, so it is also called as fibula. Before and after ligament with the ligament fibula ligament is the weakest of the ankle ligaments. (3) The inferior tibia and the inferior tibia ligament, also called the tibia and transverse ligament. There are two, respectively, at the lower end of the tibia and the posterior tibia fibula closely linked to deepen the ankle before; rear, stable ankle joint. From the perspective of ankle anatomy, ankle ligaments, fine fiber and elasticity is not easy to be injured, so most of ankle ligament injury mainly concentrated lateral 


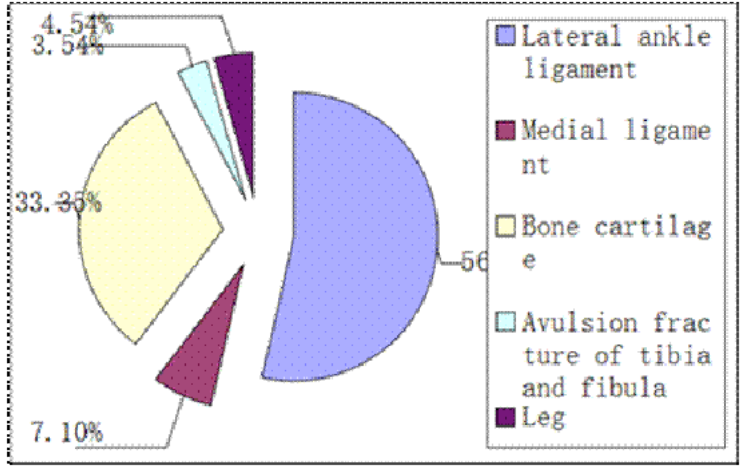

Fig. (2). Damage parts of ankle joints.

collateral ligament of fibula, posterior fibula and fibula ligament Fig. (2).

\subsection{Damage Degree of Ankle Joint in Basketball Sport [7]}

According to the sports medicine for sports injury severity classification, the injured after more than a week can not training, rest treatment for severe injury. One week after the injury in training, but need to stop or reduce the risk of activities of the department for moderate injury. If injuries can be planned normal training, and does not cause the injury is considered aggravated mild trauma

From the Table 3, the basketball sports ankle injuries in mild and moderate damage, accounted for $51.1 \%$ and $37.8 \%$, respectively, followed by severe damage, accounting for $11.1 \%$. While light, moderate injury is often easily overlooked by the injured, do not pay attention to. If the treatment is unreasonable or not timely may cause damage again, so easily cause athletes ankle joint seriously injured, and even in advance of the sports career [8].

\subsection{Damage Reason of Ankle Joint}

In the ankle injury investigation found that reason is not reasonable due to injuries accounted for $35.36 \%$; for flight landing step on someone's foot caused by $31.43 \%$ damage, due to insufficient preparation induced injury for $13.56 \%$; due to the venue, equipment unqualified injury accounted for $11.56 \%$; for other reasons guide injury accounted for $8.6 \%$ Table 4. Fig. (3) [9].

In the game of basketball mechanical reasons ankle injury statistics, injuries occur when landing accounted for

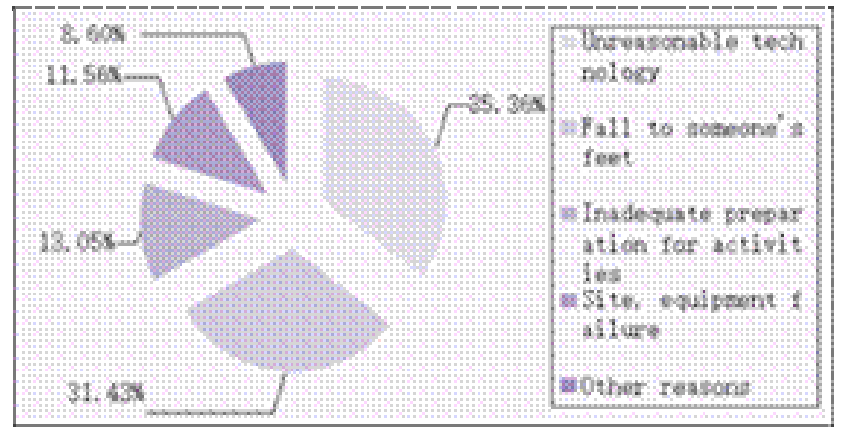

Fig (3). Injury factors of ankle joint.

$45.0 \%$ vacant, and fell on the ground of improper accounting for about half of each others feet, which is consistent with earlier studies. Other reasons were is Sharp $(30.0 \%)$, collision $(10.0 \%)$, falls $(5.0 \%)$, emergency stop (22.5\%), trips $(2.5 \%)$, others $(5.0 \%)$. basketball technique requires frequent movement off, so when damage occurs vacated landing mechanical reasons accounted for almost half the damage mechanism is vacated after athletes, ankle plantar flexion in the natural state, before the talus is wider, narrower rear portion of the ankle hole, thus ankle becomes unstable, landing posture or fall as others feet, shift the focus on one foot is more prone to injury. mechanical reasons but also with other aspects of the center of gravity shifted to focus on one foot important links lead to injury Fig. (4). Therefore, strengthening technology development of a proper diet, and strengthen self-protection awareness, grasp buffering technology to improve balance.

\subsubsection{Technical Action is Unreasonable}

Because of the shortcomings and technical violations of the technical movement of human structure function and the mechanical principle of the movement, the movement is the main reason for the damage.

\subsubsection{Tread on Someone Else After Landing [10]}

The basketball technical requirements in sports frequent jump, in the high resistance, fast paced movement, so while the flight landing, it's easy to damage. The damage mechanism is that after athletes vacated, ankle joint in natural overstep bent shape, anterior talar wide, behind the narrow section go into the ankle mortise, and ankle is closed to become unstable, that can fall on the other foot, offset of center of gravity in the foot which is more prone to damage, especially

Table 3. Injury degree of ankle joint in basketball sport.

\begin{tabular}{|c|c|c|c|}
\hline Damage Degree & Light & Moderate & Severe \\
\hline \hline Proportion (\%) & 51.1 & 37.8 & 11.1 \\
\hline
\end{tabular}

Table 4. Injury factors of ankle joint.

\begin{tabular}{|c|c|c|c|c|c|}
\hline Total Person Time & $\begin{array}{c}\text { Unreasonable } \\
\text { Technology }\end{array}$ & $\begin{array}{c}\text { Fall to Someone's } \\
\text { Feet }\end{array}$ & $\begin{array}{c}\text { Inadequate Prepara- } \\
\text { tion for Activities }\end{array}$ & $\begin{array}{c}\text { Site, Equipment } \\
\text { Failure }\end{array}$ & Other Reasons \\
\hline \hline 67920 & $35.36 \%$ & $31.43 \%$ & $13.05 \%$ & $11.56 \%$ & $8.6 \%$ \\
\hline
\end{tabular}




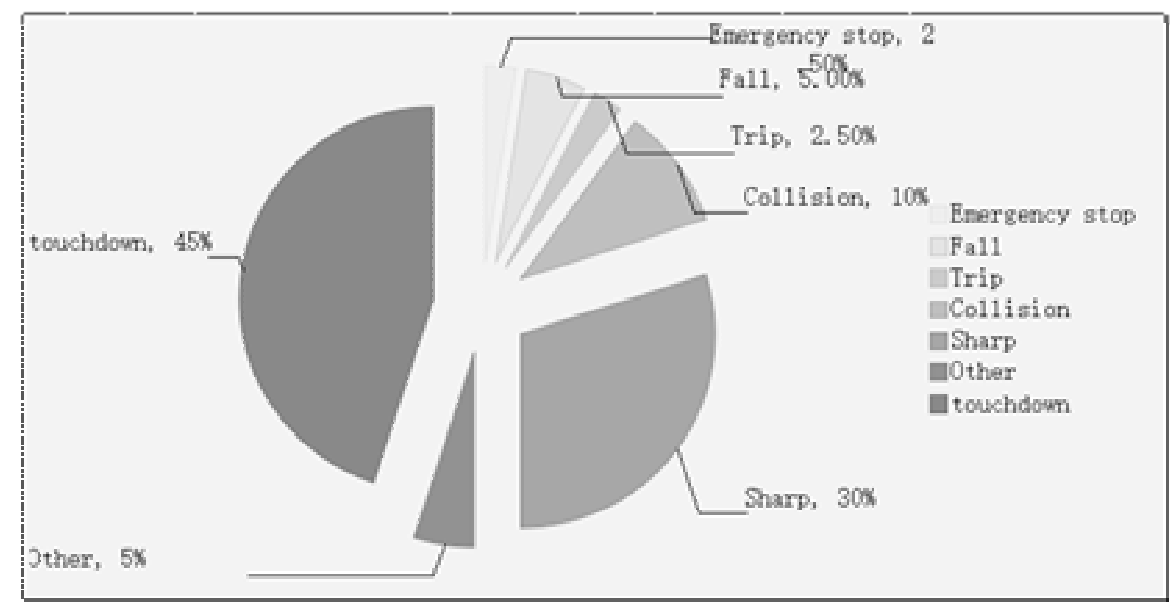

Fig (4). Mechanical reasons of motor ankle injuries.

Table 5. The timing of the injury of ankle joint movement

\begin{tabular}{|c|c|c|c|c|c|}
\hline Occurrence Timing & Rebound & Breakthrough & Defense & Shoot & Shoot \\
\hline \hline Proportion (\%) & 40 & 26.7 & 22.2 & 6.7 & 4.4 \\
\hline
\end{tabular}

when trampled players put foot jerked travel time and damage caused by serious. Landing step on someone's foot is apt to cause the ankle ligament injury that is due to ankle rely mainly on the fixation of ankle ligament and lateral ligaments. Trochlea of talus is widened, and the ankle plantar flexion cause varus and valgus in leaving the place. In the basketball training and the competition of a lot of jump, the moment, the ankle load can reach $400 \mathrm{~kg}$ or more, so it's more easily injured. In the offensive and defensive combat, with speed, requiring players step is flexible, especially the defenders. In order to occupy a favorable defensive position, footwork must be fast. In such a high resistance, fast rhythm of training and competition are very easy to make defenders in sliding or retreat step, which step on the other players on the foot and ankle injuries caused. But the damage is more than the medial and lateral malleolus. In rebounds, often using one leg, ankle joint load is increase. If the buffer is not good or when landing on the other players foot, so that their excessive plantar flexion and inversion, is prone to damage to the lateral ligament. Especially when the foot suddenly pull away from the foot players, resulting in more serious damage. Therefore, preventive exercise usually has great benefits for preventing such damage. In the training and the competition, it must use the elastic bandage or the guard ankle to carry on the fixation to the ankle joint, as far as possible to choose the professional basketball shoes [11].

\subsubsection{The Preparation is Not Full}

Preparatory activities which is not fully the same is very easy to cause the ankle injury, which will make the nervous system, respiratory and circulatory function that cannot be fully mobilized, and that motion system can not give full playing to the function of potential, such as the joint stiffness, muscle strength weak, uncoordinated movements, they are easily injured. The damage rate of winter was the highest in winter, while the lowest in summer. This is because the cold winter weather, blood circulation is slow, if the activity is not sufficient, it can cause damage.

\subsubsection{Substandard Venues and Equipment}

Uneven ground, gravel or debris, the venue is too slippery, sports equipment quality which is not good or not fixed, insurance. The facilities are not up to the requirements and can cause damage.

Also the lack of safety education and injury prevention awareness are weak, in the training not actively taking preventive measures, blind training, or anxious, does not comply with the principle of gradual and capability and damage [12].

\subsection{The Occurrence Situation of Ankle Injury in Basketball}

From the Table 5, we can know, In the basketball game, the students in the rebound when the ankle joint injury more, is for $40 \%$. This is because grab rebounds action occurred in three seconds in the region. In this region, defensive and offensive students will increase the chance of contact and collision, step on the other foot, ankle more prone to injury

\subsection{The Post Injury Statistics of Basketball After Ankle Injury}

After the injury of ankle joint in basketball, many people take the measures to save themselves: $75.7 \%$ of the ice cover, $54.1 \%$ of the compression bandage, $70.3 \%$ raise limb, no use hot compress for injury immediately after the rescue measures in injury after rescue, more than half $(56.8 \%)$ did not take further specialized medical measures in occupational physicians, $62.5 \%$ of the medical recovery, $56.3 \%$ using the physiotherapy, $0.07 \%$ subjecting to surgery. In an interview to understand, $73.0 \%$ of basketball movement participation had ankle injury experience, only $25.9 \%$ injured come after the specialized medical measures [13]. 
After an ankle sprain, you should immediately stop motion, avoid weight-bearing, ankle joint immobilization and pressure dressing and cold compress. Ice cold or ice can not be in direct contact with the skin, to avoid frostbite. Pressure dressing and cold compress can effectively reduce the ligament rupture bleeding, shorten the healing time, reduce the future organization of hematoma of scar. This is acute ankle joint sprain early the basic processing method, followed by a doctor of the ankle joint injury degree that were accurate judgment and further treatment

\section{PREVENTION OF ANKLE INJURIES IN BASKET- BALL GAMES}

\subsection{Pay Attention to the Thought}

Whether teachers, coaches, or students, athletes should recognize the importance of preventing sports injury, strengthen security, organizational discipline, moral quality and other aspects of education.

We Should Respect The Laws Of Science, Scientific and Systematic Training For Basketball Technology Actions, Strengthen the Cultivation of Correct Technical Habits, Master Buffer Technology, Improve Balance, Prevent Local Burden, The Use of Specialized Equipment [14].

\subsection{Strengthen Ankle Training}

The physiological and anatomical weakness of ankle is weak, and the joint is unstable. Only by strengthening the ankle joint exercise can we prevent the injury of ankle joint more effectively. Method of exercise ankle is: weight lifting heel and bearing the cross for jumping, ankle external rotation and internal rotation, internal oblique, concave or convex for various jumping movement practice.

\subsection{Master Reasonable Technical Movements}

Master buffer technology. Landing buffer technology is a basketball player must master the technical movements, which can defuse the impact of risk; improve the balance of power. Balance is not only a physiological reaction process of brain thinking, but also a series of systematic and orderly physiological reactions of a brain, nerve and muscle. According to the characteristics of basketball special multiple injuries, it should pay attention to the reasonable arrangement of local motion load, to avoid too much easy to hurt the action exercises to overcome "singles" training method. For example, in order to prevent the patella strain, for the special force of the knee squat and auxiliary training cannot be arranged too concentrated and too much.

\subsection{Be Prepared for Activities}

Preparation activities is to practice before training or competition, including general and special preparation activities, which can isolate motion system fully activities; improve irresolute system excitability cycle, make the body into a suitable competition state to faster adaptation fierce competition or training [15], In any competition or training, it should conscientiously do a good job in the preparatory activities The body is fever, sweating slightly, to fully open the back and limbs, joints, muscles, to effectively prevent muscle strain and sprain. In addition, the amount of preparation activity to close to the official training or competition, the time of the two can not be too long. Pay attention to the preparation of activities, in the training and the game should be fully prepared, this can improve the excitability of the body. To prepare for the activities to be effective, appropriate, targeted, especially in the history of the students with ankle injuries to prepare the joint to prepare the activity, you can avoid damage again.

According to the characteristics of basketball special multiple injuries, it should pay attention to the reasonable arrangement of local motion load, which avoid too much easy to hurt the action exercises to overcome "singles" training method. For example, in order to prevent the patella strain, the special force of the knee squat and auxiliary training cannot be arranged too concentrated and too much.

\subsection{Attention to the Choice of Venue Equipment}

We should be in the course of the basketball court and equipment safety hazards inspection, the maximum to avoid the venue equipment to bring unnecessary damage. Such as site hard surface, sliding, uneven, cracks etc.

Except afore-mentioned a few, you should pay attention to diet and nutrition, sports fatigue recovery, to ensure the conservation of energy and improve the safety awareness of athletes, strengthen medical supervision, strengthen the protection and self-protection, which can ensure the training ground to comply with the standards for physical health, but also the prevention of ankle injury is an important content of the!

\section{CONCLUSION}

In basketball, ankle injury has become the most common sports injury in basketball. The main reason is the special physiological structure, and the technical movement of the basketball players is unreasonable, the preparation is not enough or the activity is not, and the bad weather and the site is not adapted. Movement to follow after the warm-up exercise physiological responses change rule, strengthen the ankle joint training, which do the preparatory activities, rational use of technology, the technical movement to follow the principle of gradual and orderly progress, as far as possible reduction of ankle injury. Reasonable collision in the game will cause damage. So in the attack and defense confrontation, we pay attention to protect themselves. If the collision falls, the protective action should be used to prevent damage. Such as the rebound in the air of the waist and feet smooth landing support technology; running layup feet landing habits; active or being knocked down the scroll buffer; off the ball after the layup postural balance; arms elbow flexion by opening anti-collision and so on.

\section{CONFLICT OF INTEREST}

The author confirms that this article content has no conflict of interest.

\section{ACKNOWLEDGEMENTS}

Declared none. 


\section{REFERENCES}

[1] G. J. Wang, X. J. Yang, and Y. Wang, "The cause and prevention of basketball sports injury," Scientific and Technological Information (Scientific and Technological), vol. 07, pp. 201-214 2008.

[2] J. Li, and F. L. Zhang, "Recovery exercise of common sports injuries," Sports world (Academic Edition), vol. 01, pp. 105-106, 2009.

[3] P. X. Jiang, and Q. C. Zhai, "Mechanism and treatment of acute ankle injury," Sichuan Sports Science, vol. 02, pp. 10-18, 2005.

[4] C. J. Huang, "Reasons and preventive measures of basketball injury in college basketball,"Journal of Minjiang University, vol. 02, pp. 12-16, 2007.

[5] H. Sun, "Investigation and prevention of knee ankle injuries in high level basketball players in Colleges and Universities," Sichuan Sports Science, vol. 03, pp. 10-20,2005.

[6] J. L. Huang, "Cause and prevention of basketball injury in college basketball," Guangxi Teachers Education University, vol. 08, 2009.

[7] J. W. Wang, "Investigation and analysis of College Students' sports injury and its prevention and treatment," Occupational Technology, vol. 03, pp. 74-78, 2009.
[8] Q. Liu, "Reasons and preventive measures of sports injuries for young basketball players," Journal of Hubei Normal University Nature Science Edition, vol. 25, pp. 1-18, 2005.

[9] C. Jiang, "The diagnosis and treatment of sports injury in the campus out of, Chinese doctor, vol. 02, pp. 25-35,2009.

[10] B. Zhao, "Sports injury and Prevention," Guangxi normal press, 2009.

[11] Y. Fang, "Cause analysis and prevention of ankle injury in basketball game," Culture Of Science And Education, vol. 25, no. 14, pp. 101-115,2010.

[12] Z. Liang, "Cause and prevention of ankle injury in basketball sport," Theoretical Exploration, vol. 02, 2009.

[13] B. K. Yi, "Investigation and analysis of the injury of ankle in basketball training and competition," Journal of Yunmeng, vol. 20, pp. 11-20, 1999.

[14] X. J. Zhang, "Prevention of ankle injury in college basketball games," Journal Of Chengde Nationality Division, vol. 23, 2003.

[15] "Analysis on the prevention and recovery training of ankle joint strain in Basketball Players," Hubei sports science and technology, vol. 22, 2003.

Received: June 10, 2015

Revised: July 29, 2015

Accepted: August 15, 2015

(C) Zhixiang Jin; Licensee Bentham Open.

This is an open access article licensed under the terms of the (https://creativecommons.org/licenses/by/4.0/legalcode), which permits unrestricted, noncommercial use, distribution and reproduction in any medium, provided the work is properly cited. 